\title{
TECHNICAL ANALYSIS BASED ON PRICE-VOLUME SIGNALS AND THE POWER OF TRADING BREAKS
}

\author{
FRANK H. WESTERHOFF \\ University of Osnabrueck, Department of Economics \\ Rolandstrasse 8, D-49069 Osnabrueck, Germany \\ fwesterho@oec.uni-osnabrueck.de
}

Received 25 January 2005

Revised 17 June 2005

\begin{abstract}
We propose a novel stock market model and investigate the effectiveness of trading breaks. Our nonlinear model consists of two types of traders: while fundamentalists expect prices to return towards their intrinsic values, chartists extrapolate past price movements into the future. Moreover, chartists condition their orders on past trading volume. The model is able to replicate several stylized facts of stock markets such as fat tails and volatility clustering. Using the model as an artificial stock market laboratory we find that trading breaks have the power to reduce volatility and - if fundamentals do not move too strongly — also mispricing.
\end{abstract}

Keywords: Stock market dynamics; technical and fundamental analysis; stylized facts; market efficiency; trading breaks; computer experiments.

\section{Introduction}

Stock markets are highly volatile and frequently display severe bubbles and crashes [32]. The question therefore arises as to what drives stock market dynamics. The arrival of new information, naturally, has an impact on stock prices. However, changes in fundamentals hardly account for all the variability observed in stock prices. The chartist-fundamentalist approach $[3,5,6,10,17,23]$ asserts that the trading behavior of boundedly rational market participants also has a significant influence on stock prices. In these models, heterogeneous agents apply technical and fundamental analysis to determine their orders. Technical trading rules rely on past movements of the stock price as an indicator of market sentiment and extrapolate these into the future, thus adding a positive feedback to the dynamics. Fundamental trading rules are designed to exploit differences between prices and fundamentals. Fundamentalists who trade on a reduction of the mispricing add a negative feedback to the dynamics. Interactions between these types of trading rules may create complex nonlinear dynamics such as temporary dependence in volatility. In general, these models are quite successful in replicating the stylized facts of financial markets. 
The goal of this paper is twofold. First, we develop a novel stock market model in which the orders of the chartists are not only based on past prices (as in the aforementioned models) but also on past trading volume. Popular technical analysts such as Murphy [27] argue that volume provides an important confirmation signal of price action: increasing volume indicates a robust trend whereas decreasing volume indicates a weakening trend. Within our model, such price-volume based technical trading strategies may cause intricate nonlinear dynamics. Suppose, for instance, that trading volume is high. Then the chartists perceive rather strong trading signals. Since they submit a high number of orders, trading volume and volatility tend to remain high. Overall, our model is able to reproduce many of the complex phenomena observed in real stock markets, including bubbles and crashes, excess volatility, fat tails for the distribution of the returns, uncorrelated price changes, and volatility clustering.

Second, we use our setup as an artificial laboratory to explore the consequences of trading breaks. Trading breaks interrupt the trading process when prices are about to exceed a pre-specified limit. We find that trading breaks have the power to stabilize financial markets: both deviations from fundamentals and price volatility may decline. In brief, trading breaks work as follows. By limiting daily price changes, volatility is directly reduced. Furthermore, price-generated technical trading signals are destroyed. Finally, since trading breaks also reduce trading volume, the remaining technical trading signals appear to be less trustworthy.

Whether or not trading breaks improve market efficiency is still an open question [12]. One reason is that conclusions from pure empirical studies are difficult to draw. For instance, without knowing the fundamental value of an asset, excess volatility and mispricing cannot be measured precisely. We therefore follow a different track and conduct computer experiments. If an artificial stock market model such as ours is properly calibrated, computer experiments may help us to understand the workings of certain regulatory mechanisms. Moreover, one can measure fundamentals accurately, control for all kinds of shocks and generate as many observations as required. The results presented in this paper support previous findings by Westerhoff [34]. In his framework, traders switch between technical and fundamental analysis depending on conditions such as market historical volatility. Trading breaks may thus calm down markets since they promote the use of fundamental analysis. For related approaches concerning transaction taxes or central bank interventions see Ehrenstein [8], Westerhoff [35] or Wieland and Westerhoff [37].

The paper continues as follows. Section 2 reviews empirical evidence concerning the agents' trading behavior. Section 3 presents the model and Sec. 4 illustrates its dynamics. Section 5 explores the consequences of trading breaks and Sec. 6 concludes the paper.

\section{The Behavior of Chartists and Fundamentalists}

Many psychological experiments support the notion of bounded rationality. As early as 1955, Simon [29] argued that people lack the cognitive capability to derive fully 
optimal actions. However, this does not imply that agents are irrational. In fact, agents strive to do the right thing. Empirical evidence indicates that agents tend to rely on a limited number of heuristic principles which have proven to be useful in the past [16].

In the case of financial markets we have solid information about the agents' trading rules. Asset pricing experiments [30, 31] reveal that agents use simple extrapolative and regressive forecast methods to predict prices. Moreover, questionnaire studies - carried out in different countries at different times - inform us that professional traders rely on technical and fundamental analysis to determine their investment positions [20, 26, 33]. For short run predictions, both concepts appear to be equally important.

Let us briefly sketch the key elements of these trading strategies. Fundamental analysis is the study of economic, industrial, and corporate conditions in an effort to determine the intrinsic (fundamental) value of a company's stock. More precisely, a fundamentalist aims at estimating the expected future cash flow of a company and uses projections of interest rates to assess the present value of the cash flow. For a fundamentalist, the market price of a stock tends to move towards its intrinsic value. Thus the idea is to buy stocks that are undervalued and sell them when they are overvalued. From a classic economic point of view, such behavior appears to be reasonable.

Rather than attempting to compute intrinsic values, chartists instead look for price patterns that may indicate a company's future performance. As early as in the late 1880s, Charles Dow formulated the basic tenets of technical analysis. Although technical analysis has been included in financial practices for many decades, it has not yet received much academic attention. To the contrary, it is often associated with the term "voodoo finance" [19]. Standard manuals of technical analysis include Edwards and Magee [7], Pring [28] and Murphy [27]. Technical analysis is a prediction method founded in the belief that current prices represent all necessary information about an asset. Studying price action is thus all that is required. The most basic concept of technical analysis is that markets have a tendency to follow trends, hence the saying "the trend is your friend". In general, technical trading rules suggest buying when prices increase, and vice versa.

Many economists are skeptical of the usefulness of technical analysis, yet empirical work indicates that at least some technical analysis rules have been profitable in the past $[1,18,19]$. The profitability of technical analysis is an interesting issue but what is more important is the observation that many professional traders are indeed using these strategies and thereby influence the evolution of stock prices. Guided by empirical evidence, the chartist-fundamentalist approach has successfully modeled the impact of heterogeneous interacting traders on financial market dynamics. Within these models, the orders of chartists typically depend only on past price action. The novel idea of this paper is to enrich the behavior of chartists by taking price-volume signals into account.

According to chartists, the analysis of trading volume is very important. Trading volume provides clues as to the intensity of a given price trend. Low-volume levels 
are characteristic of the indecisive expectations that typically occur during consolidation periods, i.e., periods in which prices move sideways. High-volume levels are characteristic of persistent price trends when there is a strong consensus that prices will move further in their current direction.

The chartist's explanation of why volume determines the health of an existing trend is as follows: rising prices coupled with high volume signifies increased upside participation (more buyers) that should lead to a continued move, whereas falling prices coupled with high volume signifies increased downside participation (more sellers). Conversely, price trends accompanied by low volume are suspect. Whether or not the logic behind this reasoning is correct is not crucial. What matters is that chartists act in accordance with this directive.

\section{A Simple Stock Market Model}

In this section, we propose a model in which fundamentalists and chartists may invest in the stocks of one company. ${ }^{1}$ Before we start describing the details of the market, let us briefly comment on our modeling approach. Our goal is to replicate a few basic qualitative properties of stock markets using a minimum set of underlying assumptions. We consider it to be important to keep the framework as simple as possible to enhance the understanding of financial market dynamics. In addition, the underlying assumptions should be in line with empirical observations. We therefore try to approximate the behavior of the market players on the basis of the evidence reported in the previous section.

Following Lux and Marchesi [23], the evolution of the fundamental value of the stock market is modeled as a random walk without drift. To be precise, the fundamental value $F$ changes with respect to the arrival of new information

$$
\frac{F_{t+1}-F_{t}}{F_{t}}=\eta_{t}
$$

where the news $\eta$ is drawn from a normal distribution with mean zero and (constant) standard deviation $\sigma^{\eta}$. Note that i.i.d. shocks add no particular structure to the dynamics, i.e., they cannot be responsible for stylized facts such as volatility clustering.

The stock price is determined on an order-driven market according to a simple price impact function [10]. A price impact function describes the relation between the number of stocks bought or sold in a given time interval and the price change caused by these trades. For simplicity, we assume a proportional relation between changes in the price $P$ and excess demand

$$
\frac{P_{t+1}-P_{t}}{P_{t}}=a\left(D_{t}^{F}+D_{t}^{C}\right) \text {. }
$$

\footnotetext{
${ }^{1}$ Restricting attention to one risky asset is a common simplification [13]. For multi-asset market models with chartists and fundamentalists see Westerhoff [36] and Chiarella et al. [4].
} 
The orders of fundamentalists and chartists are denoted by $D^{F}$ and $D^{C}$, respectively, and $a$ is a positive price impact coefficient. Accordingly, excess buying drives the stock price up and excess selling drives it down. ${ }^{2}$

Fundamentalists have a view of the intrinsic value of the stock market and consequently buy stock if they decide it is undervalued and sell stock if they decide it is overvalued. The orders of the fundamentalists may be written as

$$
D_{t}^{F}=b \frac{F_{t}-P_{t}}{P_{t}},
$$

where $b$ is a positive reaction coefficient. In agreement with the literature, fundamentalists perceive the fundamental value correctly [5]. For an interesting exception to this standard assumption see de Grauwe et al. [6].

Technical analysis is a general heading for a myriad of trading strategies. There are probably as many methods of combining and interpreting the various techniques as there are chartists themselves. However, all chartists rely on the notion of market momentum and apply some sort of positive feedback rules. The orders of chartists are formalized as

$$
D_{t}^{C}=\left(c \frac{P_{t}-P_{t-M}}{P_{t-M}}+\delta_{t}\right)\left(\frac{1}{M} \sum_{m=1}^{M} V_{t-m}\right)^{d} .
$$

The first term in the first bracket describes the chartists' trend extrapolation behavior: chartists go long (short) when the price today is higher (lower) than the price $M$ periods ago. The reaction coefficient $c$ is positive. The second term in the first bracket reflects additional random orders and thus accounts for the large variety of technical trading rules. $\delta$ is a normally distributed random variable with mean zero and constant standard deviation $\sigma^{\delta}$.

A novel aspect of this model is to condition the orders of the chartists on past trading volume. Chartists argue that trading volume represents a measure of the intensity of a price trend. The greater the volume, the more chartists expect the existing trend to continue rather than to reverse. Trading volume is defined as the sum of the absolute amount of the orders of chartists and fundamentalists, i.e.,

$$
V_{t}=\left|D_{t}^{F}\right|+\left|D_{t}^{C}\right|
$$

The second term of (3.4) thus implies that the higher the average trading volume of the last $M$ trading periods, the more forcefully the chartists act on their trading signals. The positive exponent $d$ captures the relevance of the trading volume for the strength of the trading signals. Note that for $d=0$, trading volume is irrelevant for the interpretation of the price signal. For $0<d<1$, the curvature of (3.4), with respect to an increase in the trading volume, is concave and for $d>1$ it is convex.

\footnotetext{
${ }^{2}$ One may interpret (3.2) as a stylized description of the behavior of market makers. Market makers then would supply excess demand from their inventory or accumulate inventory when there is an excess supply, see, e.g., Lux [21].
} 


\section{Simulation Analysis}

The price evolution equation, obtained by combining (3.1)-(3.5), is a multidimensional nonlinear stochastic difference equation. Since it precludes closed analysis, we proceed with numerical analysis. Note that it is quite simple to simulate the dynamics of the model and thus to replicate and check the robustness of our results. Throughout the simulation, unless stated otherwise, we use the following parameter setting:

$$
a=1, b=0.02, c=0.04, d=0.8, M=5, \sigma^{\eta}=0.01 \text { and } \sigma^{\delta}=0.7 .
$$

In Sec. 4.1, we first illustrate the workings of the model. Section 4.2 then explores the extent to which the model is able to mimic the statistical features of real stock markets.

\subsection{The workings of the model}

Figure 1 shows the dynamics for 250 observations. From top to bottom, the panels present the development of the price (the course of the fundamental value is indicated by the dashed line), relative price changes, the orders of fundamentalists, the orders of chartists and the trading volume, respectively. Note that between $t=50$ and $t=200$ the price deviates from its fundamental value. Initially the stock is undervalued but then the price strongly exceeds its fundamental value. The price pattern is obviously reminiscent of a bubble path. During the price increase, relative price changes are higher than usual. This, of course, is triggered by the orders of the market participants. Chartists in particular are very active during this period. The reason is that chartists perceive a lasting price trend which is furthermore supported by high trading volume. This process is persistent: High trading volume stimulates orders of chartists which in turn cause high trading volume. As a result, volatility is also elevated. The orders of the fundamentalists simply depend on the magnitude of the distortion in the market. The resulting mean reversion pressure eventually pushes prices back towards their fundamentals.

\subsection{The statistical features of the model}

In recent years, it has become clear that the price statistics across a wide range of quite different financial markets exhibit certain universal properties. For indepth accounts of these stylized facts see, e.g., Mantenga and Stanley [25], Lux and Ausloos [24], Johnson et al. [15] or Sornette [32]. With respect to our study, the most important features are:

- Financial markets regularly produce bubbles and crashes. If prices always reflected their fundamental values, crashes ought to correspond to really bad shocks. However, thorough ex-post analyses of crashes are in many cases inconclusive as to what this dramatic piece of new information might have been. Empirical 

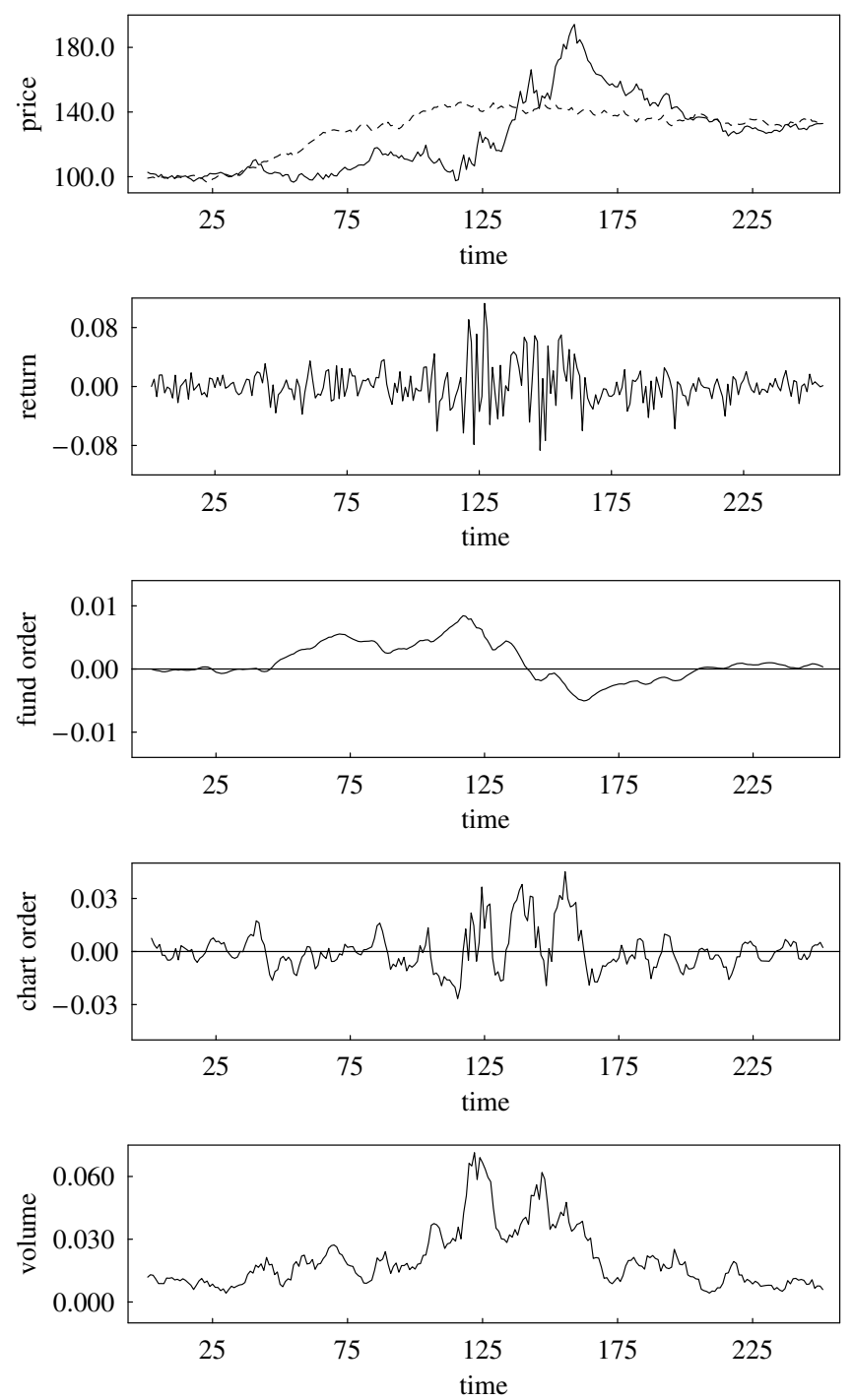

Fig. 1. The dynamics in the short run. The first, second, third, fourth and fifth panels display the evolution of the price (the fundamental value is given by the dashed line), relative price changes, orders of the fundamentalists, orders of the chartists and the trading volume, respectively. To increase visibility, the latter three variables are plotted as moving averages using five lag periods. Parameter setting as in Sec. 4; 250 observations.

studies suggest that the majority of crashes occur endogenously due to market instabilities.

- Price volatility is too high to be justified by fundamental shocks. As just mentioned, distinct price changes often appear to be unrelated to the arrival of significant new information. Excess volatility is also reflected in the fact that the price 
of a financial asset typically moves on a second-to-second basis whereas news hits the market less frequently.

- The distribution of the returns deviates significantly from the normal distribution and possesses fat tails. Fat-tailedness may be quantified by estimating the socalled tail index. For many different assets, the tail index hovers between three and four, implying that the fourth moment of the distribution of the returns (i.e., the kurtosis) does not exist.

- Price increments are uncorrelated. For instance, the autocorrelation function of raw returns is essentially zero for all time lags. Moreover, Hurst exponents of around 0.5 indicate the absence of memory in the return process.

- Periods of low volatility alternate with periods of high volatility. The autocorrelation function for absolute returns is positive and decays slowly. Temporal correlation in volatility may last several months. Hurst exponents for absolute returns are usually above 0.6 , which again implies persistent behavior.

Let us now explore whether the statistical features of the simulated dynamics are in harmony with the stylized facts of actual financial markets. Figure 2 is designed to provide a first answer. The first panel presents the distortion in the time domain, i.e., the relative deviation of the price from its fundamental value. As can be seen, strong and lasting bubbles emerge. The distance between the stock price and its fundamental value may be larger than $30 \%$.

The second panel shows relative price changes of the stock (i.e., the returns), and the third panels depicts relative changes in the fundamental value (i.e., the news). Extreme returns may be as large as 10\%, while the impact of new information on the fundamental value is much smaller. Within our simulations, volatility is obviously excessive. Remember that news is i.i.d. normal. Visual inspection reveals that this is clearly not the case for stock returns.

The bottom two panels display the autocorrelation function of raw returns and of absolute returns, respectively. The dotted lines indicate $95 \%$ confidence bands. Note that for almost all lags, the autocorrelation of raw returns is not significant. Hence, the evolution of stock prices resembles a random walk. A different picture emerges when one explores the autocorrelation function of absolute returns. The autocorrelation coefficients for the first 50 lags are positive and significant, a clear sign of volatility clustering. ${ }^{3,4}$

Let us ponder the implications of this outcome. If prices always matched their fundamental values, then the fat tail property would merely be a reflection of the probability distribution of the exogenous fundamental shocks hitting the market. Moreover, volatility clustering would arise since the intensity of news alternates over time. Although the news arrival process is constructed as a random walk process within our setup, non-random-walk price behavior emerges as a result of

\footnotetext{
${ }^{3}$ For $M=1$, the law of motion of the stock price is of the fifth order. Nevertheless, temporal dependence in volatility arises with significant autocorrelation coefficients up to about 25 lags. ${ }^{4}$ In addition, real markets display a strong cross-correlation between trading volume and volatility [2]. This phenomenon is also observed within our simulations.
} 

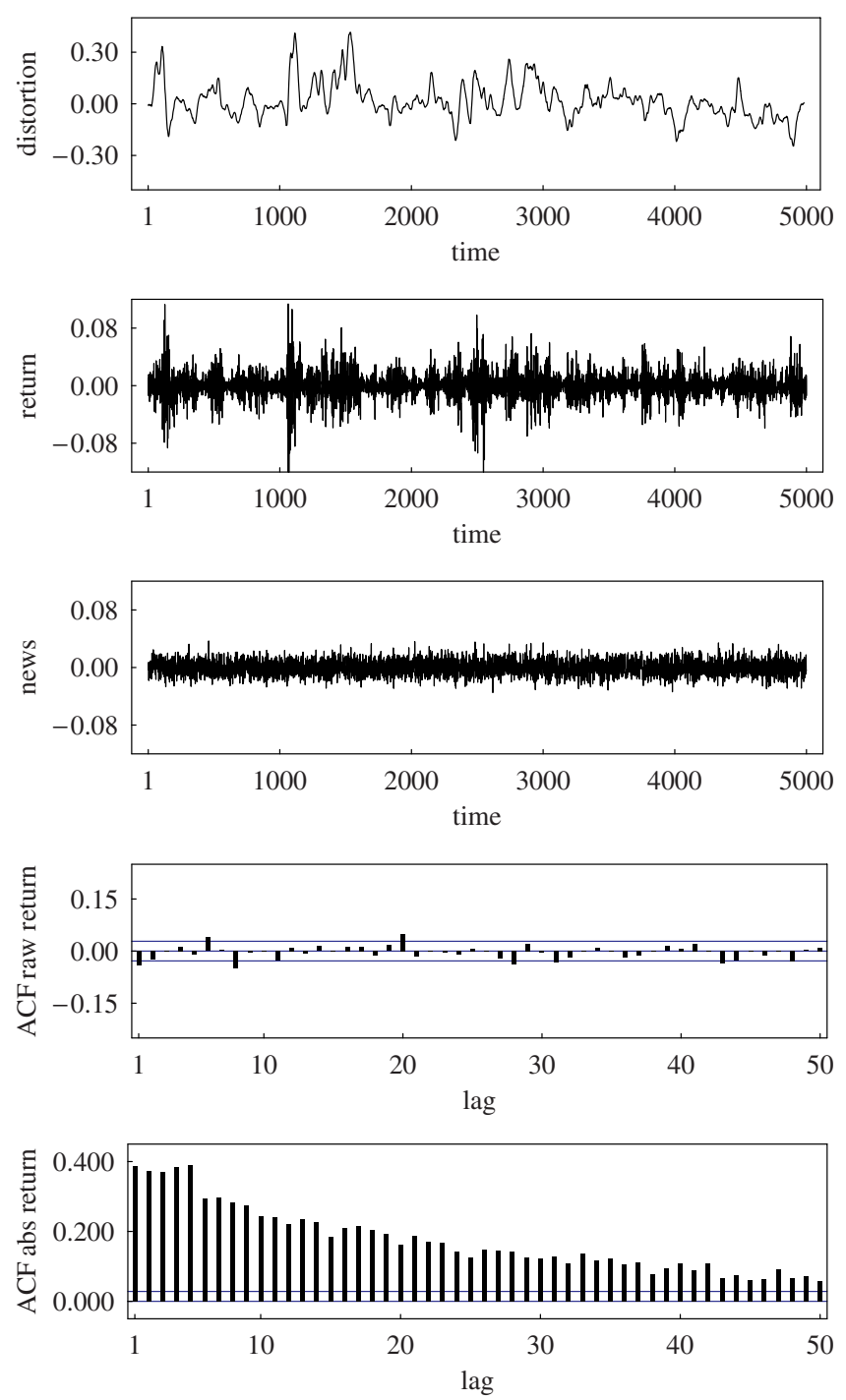

Fig. 2. The dynamics in the long run. The first, second, third, fourth and fifth panels display relative deviations between prices and fundamentals, relative price changes, relative changes in fundamentals, the autocorrelation function of raw returns and the autocorrelation function of absolute returns. The dotted lines in the bottom two panels indicate 95\% confidence bands. Parameter setting as in Sec. 4; 5,000 observations.

the nonlinear interaction between the market players. This shows that one does not need to assume a complex information flow to account for the intricate behavior of financial prices. Clearly, the stylized facts are endogenously caused through the trading process itself.

Figure 2 illustrates the dynamics for a single time series. In order to check the robustness of our findings, Table 1 provides some key statistics for 11 simulation 
Table 1. Summary of stylized facts. The table reports estimates for the distortion, the volatility, the tail index, the Hurst exponent for raw returns and the Hurst exponent for absolute returns, respectively. The 11 simulation runs are generated with different seeds of random variables. Each run contains 5,000 observations. Parameter setting as in Sec. 4.

\begin{tabular}{cccccc}
\hline Run \# & Distortion & Volatility & Tail index & Hurst return & Hurst |return| \\
\hline 01 & 0.077 & 0.013 & 3.469 & 0.531 & 0.663 \\
02 & 0.078 & 0.014 & 3.278 & 0.524 & 0.658 \\
03 & 0.092 & 0.015 & 3.436 & 0.520 & 0.665 \\
04 & 0.080 & 0.014 & 3.000 & 0.530 & 0.667 \\
05 & 0.093 & 0.016 & 3.291 & 0.515 & 0.673 \\
06 & 0.084 & 0.014 & 3.640 & 0.526 & 0.670 \\
07 & 0.084 & 0.015 & 3.022 & 0.530 & 0.666 \\
08 & 0.075 & 0.014 & 3.363 & 0.534 & 0.668 \\
09 & 0.085 & 0.014 & 3.094 & 0.522 & 0.665 \\
10 & 0.085 & 0.014 & 3.066 & 0.538 & 0.669 \\
11 & 0.103 & 0.016 & 2.610 & 0.521 & 0.659 \\
\hline
\end{tabular}

runs, each containing 5,000 observations. The time series are generated with the same parameter setting but with different seeds of random variables. The results are striking. The distortion, defined as the average absolute relative distance between the stock price and its fundamental value, ranges between $7.5 \%$ and $10.3 \%$, with $8.4 \%$ as the median value. Volatility, computed as the average absolute relative price change, varies between $1.3 \%$ and $1.6 \%$. In comparison, the average absolute relative change of the fundamental value is about $0.8 \%$. Price volatility may thus be twice as high as warranted by fundamentals.

We apply the Hill tail index estimator to compute the tail index [22]. Using the largest $5 \%$ of the observations, tail indices between 3.61 and 3.64 are derived. Such estimates correspond well with estimates obtained from actual stock markets. The last two columns report Hurst exponents, calculated with the rescaled range method [14]. Hurst exponents for raw returns hover between 0.515 and 0.538. Roughly speaking, Hurst exponents which fall within the range of about 0.45 and 0.55 may be interpreted as representative of random walk processes. Hence, we again have hints that the simulated price increments are fairly uncorrelated. Hurst exponents for absolute returns scatter between 0.658 and 0.673 . Such values imply strong persistence in volatility. Absolute returns of actual stock markets are typically above 0.6. Again, the model produces statistics which are comparable with real markets.

The key parameter of our model is the parameter $d$ which captures the relation between the chartists' price and volume signals. Let us thus briefly explore how $d$ affects the dynamics of the model. Table 2 presents minimum, median and maximum estimates of the distortion, the volatility, the tail index, the Hurst exponent for raw returns and the Hurst exponent for absolute returns, respectively, for $d \in\{0.0,0.1,0.2,0.3,0.4,0.5,0.6,0.7,0.8,0.9,1.0\}$. For $d=0$, raw returns are uncorrelated but neither do we observe fat tails for the distribution of the returns nor volatility clustering. As $d$ increases, the dynamics becomes more interesting. 
Table 2. Robustness of the results. The table reports median estimates for the distortion, the volatility, the tail index, the Hurst exponent for raw returns and the Hurst exponent for absolute returns, respectively, for different values of $d$ (minimum and maximum estimates of the statistics are given in brackets). The same simulation design as in Table 1.

\begin{tabular}{cccccc}
\hline$d$ & Distortion & Volatility & Tail index & Hurst return & Hurst $\mid$ return| \\
\hline 0.0 & 0.720 & 0.112 & 6.302 & 0.514 & 0.526 \\
& $(0.615-0.842)$ & $(0.110-0.115)$ & $(5.660-6.598)$ & $(0.505-0.530)$ & $(0.519-0.545)$ \\
0.1 & 0.667 & 0.105 & 6.285 & 0.515 & 0.549 \\
& $(0.567-0.778)$ & $(0.103-0.108)$ & $(5.332-6.472)$ & $(0.501-0.531)$ & $(0.531-0.558)$ \\
0.2 & 0.608 & 0.098 & 5.963 & 0.516 & 0.554 \\
& $(0.513-0.707)$ & $(0.096-0.101)$ & $(5.426-6.483)$ & $(0.503-0.532)$ & $(0.544-0.573)$ \\
0.3 & 0.541 & 0.089 & 5.607 & 0.517 & 0.569 \\
& $(0.453-0.626)$ & $(0.087-0.092)$ & $(5.257-5.887)$ & $(0.505-0.534)$ & $(0.559-0.588)$ \\
0.4 & 0.465 & 0.079 & 5.291 & 0.519 & 0.587 \\
& $(0.387-0.535)$ & $(0.076-0.082)$ & $(4.879-5.729)$ & $(0.506-0.535)$ & $(0.576-0.604)$ \\
0.5 & 0.375 & 0.066 & 4.700 & 0.522 & 0.603 \\
& $(0.313-0.431)$ & $(0.063-0.069)$ & $(4.585-5.446)$ & $(0.508-0.536)$ & $(0.594-0.620)$ \\
0.6 & 0.277 & 0.050 & 4.342 & 0.524 & 0.622 \\
& $(0.233-0.328)$ & $(0.048-0.054)$ & $(4.040-4.857)$ & $(0.511-0.537)$ & $(0.612-0.638)$ \\
0.7 & 0.176 & 0.032 & 3.745 & 0.524 & 0.644 \\
& $(0.151-0.216)$ & $(0.030-0.036)$ & $(3.388-4.187)$ & $(0.514-0.538)$ & $(0.637-0.657)$ \\
0.8 & 0.084 & 0.014 & 3.278 & 0.526 & 0.666 \\
& $(0.075-0.103)$ & $(0.013-0.016)$ & $(2.610-3.640)$ & $(0.515-0.538)$ & $(0.658-0.673)$ \\
0.9 & 0.044 & 0.003 & 2.854 & 0.553 & 0.675 \\
& $(0.039-0.046)$ & $(0.003-0.004)$ & $(2.199-3.188)$ & $(0.547-0.565)$ & $(0.667-0.686)$ \\
& 0.039 & 0.001 & 3.012 & 0.647 & 0.681 \\
& $(0.030-0.043)$ & $(0.001-0.001)$ & $(2.452-3.356)$ & $(0.636-0.652)$ & $(0.668-0.690)$ \\
\hline
\end{tabular}

Due to the interaction between technical price and volume signals, the distribution of the returns starts to deviate from the normal distribution and temporal correlation in volatility sets in. For about $d=0.8$, the model best matches the stylized facts of financial markets. If $d$ increases further, unrealistic correlations in raw returns emerge.

To summarize, our model has the potential to match some basic features of stock markets. At least some popular statistical tests do not discriminate between actual and artificial data. The stylized facts are almost completely due to the interactions between the agents. The behavior of the chartists is on average destabilizing, thus they tend to drive the price away from its fundamental value. However, the distortion is countered by the orders of the fundamentalists. Excess volatility is mainly caused by the chartists. Since they use an ocean of different technical trading rules, a substantial amount of their orders are stochastic. As a result, the price evolution appears as a random walk. Fat tails are triggered by the nonlinear demand function of the chartists: extreme price changes result when trading volume is high, and vice versa. This mechanism is also responsible for volatility clustering. High trading volume stimulates orders of chartists which cause high volatility. Both volatility and trading volume are likely to remain elevated. Despite the model's minimal set of 
assumptions and parameters, its statistical features are in good agreement with the stylized facts observed in real financial markets. We thus think that it is appropriate to continue our analysis and investigate the effectiveness of trading breaks.

\section{The Power of Trading Breaks}

Volatile and distorted stock markets entail large risks to investors. Regulators may therefore impose restrictions on the trading process. The aim of this section is to use our stock market model as a test bed to explore the effectiveness of trading breaks. Trading breaks, also called circuit breakers or price limits, work as follows: if the movement in the stock price exceeds certain bounds, then trading is automatically interrupted. Regulators hope that in subsequent periods trading will be calmer. For instance, trading breaks may give nervous investors time to cool off and to reassess their information. Trading breaks are used in many stock markets around the world, including France, Italy, Japan, Korea, Switzerland, Taiwan and the United States. For surveys on this topic see France et al. [11] or Harris [12].

In the following simulations we now restrict the price adjustment to

$$
P_{t}(1-i)<P_{t+1}<P_{t}(1+i),
$$

where $i$ indicates the maximum allowed price change per period. For example, if $i=0.05$ then prices may increase or decrease at most $5 \%$ from their previous period's value. If the price hits the upper or lower limit, trading is stopped for that period.

The traders submit their orders as expressed in (3.3) and (3.4). However, if the trading process is interrupted, not all orders can be executed. Thus we rescale the trading volume as

$$
V_{t}=X_{t}\left(\left|D_{t}^{F}\right|+\left|D_{t}^{C}\right|\right)
$$

where the scaling factor $X$ is given as

$$
X_{t}= \begin{cases}\left|\frac{i}{\left(P_{t+1}^{\prime}-P_{t}\right) / P_{t}}\right|, & \text { for } \frac{\left|P_{t+1}^{\prime}-P_{t}\right|}{P_{t}}>i, \\ 1, & \text { otherwise. }\end{cases}
$$

$P^{\prime}$ is defined as the price that would have occurred without a price limit. Suppose, for instance, that the price limit is $i=5 \%$ and that the price change without the price limit would have been $10 \%$. Then the scaling factor is $X=0.5$, i.e., only half of the traders' orders are filled. If the price limit is not hit, all orders are executed and $X=1$. The underlying idea of (5.2) and (5.3) is that the trading volume and the actual price change are proportional.

Let us explore how trading breaks affect the dynamics of the model. Figure 3 contains a simulation run where regulators have imposed a price limit of two percent. Figure 3 can directly be compared with Fig. 1 since it is based on the same seed of random variables. What are the differences? The first panel reveals that the 

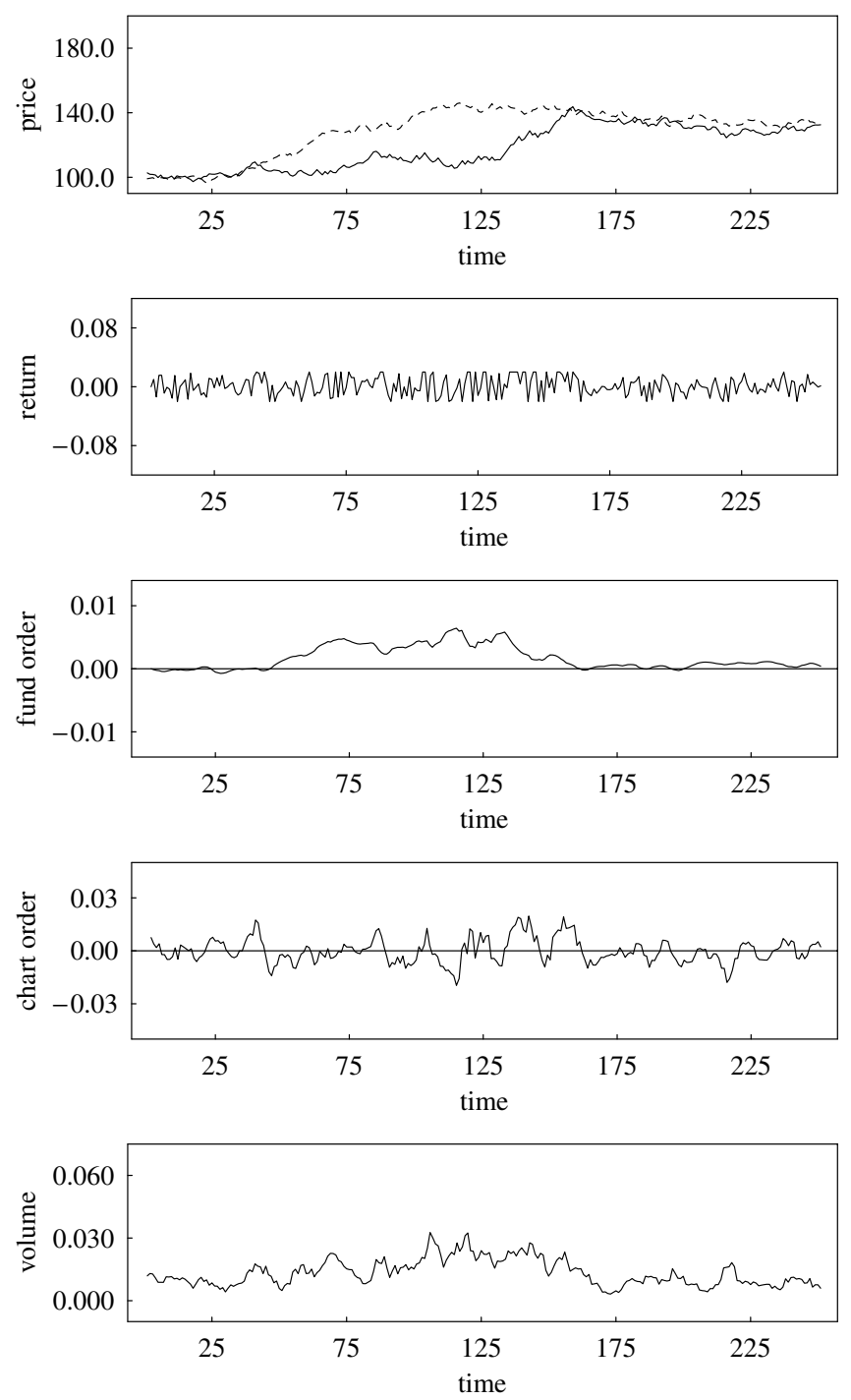

Fig. 3. The impact of price limits. The same simulation design as in Fig. 1 but now with a price limit of $2 \%$.

previous bubble has almost vanished, i.e., mispricing has considerably improved. Trading breaks therefore do not only prevent crashes, they may also hinder bubbles from occurring in the first place - an aspect which is often overlooked when trading breaks are evaluated. The second panel shows that relative price changes do not, of course, exceed the $2 \%$ level. As a result, the stock market appears to be less risky. Note furthermore that the orders of fundamentalists and chartists as well as the total trading volume are now of a lower magnitude. Figure 3 thus suggests that price limits may stabilize stock markets. 

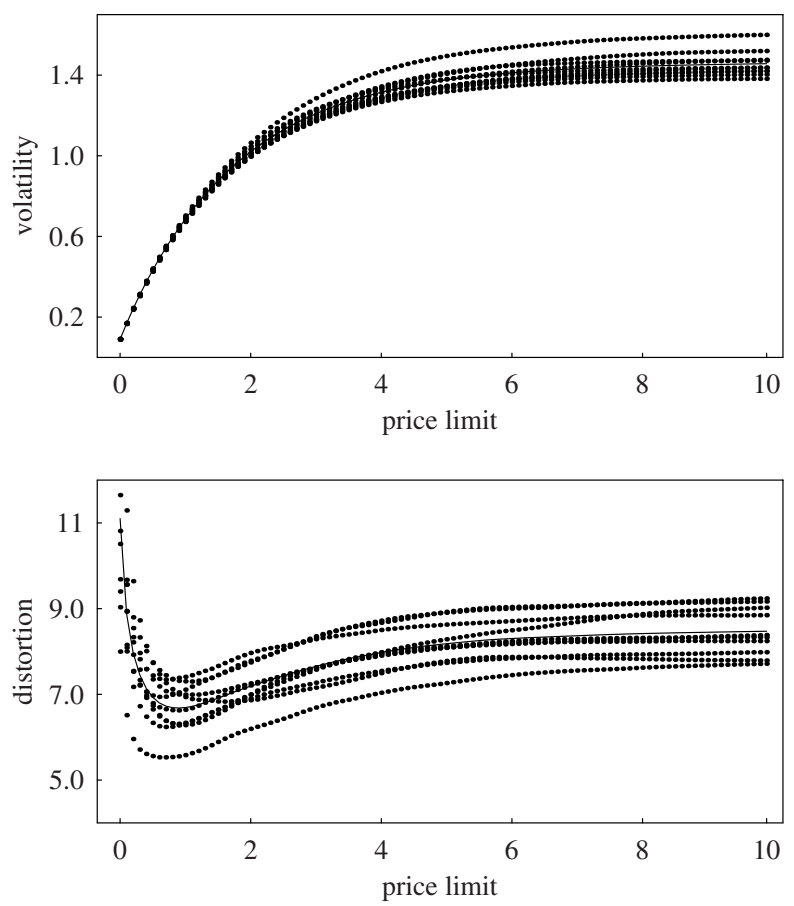

Fig. 4. The power of price limits. Price limits range between $0 \%$ and $10 \%$, increasing in 100 steps. Volatility and distortion are calculated from 5,000 observations. Estimates for ten simulation runs are displayed (different seeds of random variables). The solid lines indicate the averages. Parameter setting as in Sec. 4.

However, a more detailed analysis is needed to evaluate the usefulness of trading breaks. In Fig. 4, volatility (top) and distortion (bottom) are plotted as functions of price limits. Price limits are increased from $0 \%$ to $10 \%$ in 100 discrete steps. Each time, volatility and distortion are calculated from 5,000 observations. This procedure is repeated for 10 different seeds of random variables. The solid lines indicate the averages. The top panel shows that the sharper the price limit, the lower the volatility. The relation between price limits and distortion, however, is nontrivial. Taking our estimates literally, we observe a local minimum in the distortion for a price limit of about $1.2 \%$.

Trading breaks are successful for several reasons. By definition, trading breaks exclude price changes larger than the imposed price limit. In addition, pricegenerated technical trading signals are weakened. The remaining technical trading signals appear to be even less reliable. Since trading breaks also reduce the trading volume, price trends are regarded as less sound. All in all, the destabilizing impact of chartists is reduced. Stock prices stop tracking their fundamental values if the fundamentalists' mean reversion pressure is hindered too much. Efficient markets obviously need some price flexibility, else the distortion increases.

Advocates of the efficient market hypothesis claim that trading breaks only slow down the price discovery process [9]. Figure 5 compares the effectiveness of 

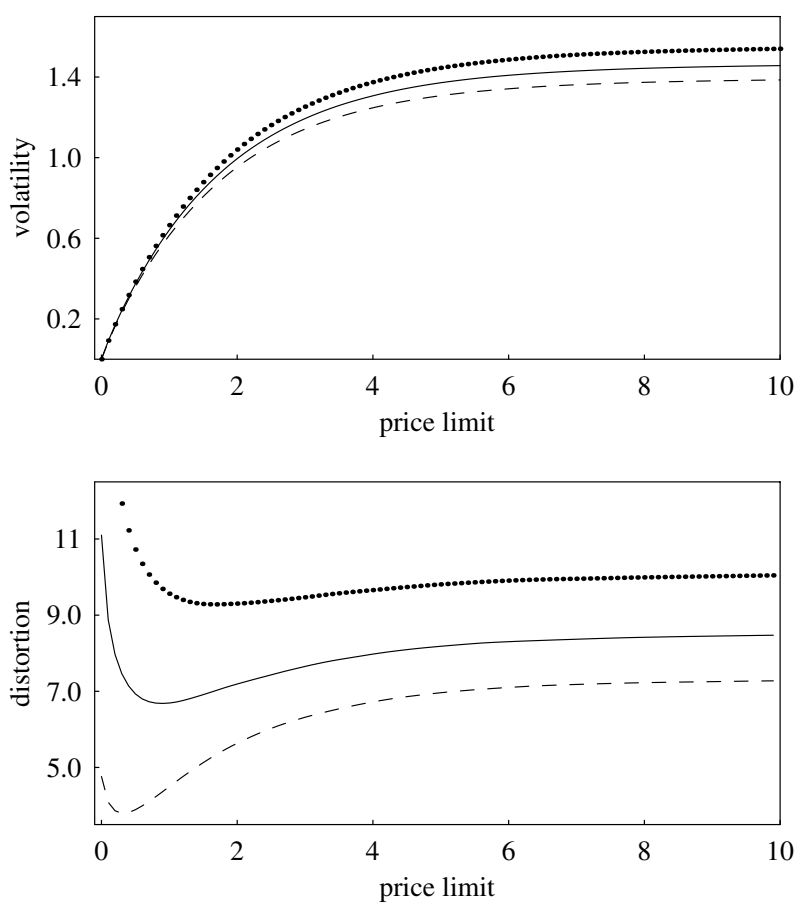

Fig. 5. Price limits and volatility in fundamentals. Volatility and distortion are plotted for increasing price limits (average values of ten simulation runs, computed in the same way as the solid lines in Fig. 4). Parameter setting as in Sec. 4, but for the solid line $\sigma^{\eta}=0.010$, for the dashed line $\sigma^{\eta}=0.005$ (low fundamental volatility regime), and for the dotted line $\sigma^{\eta}=0.015$ (high fundamental volatility regime).

price limits for three different news processes (dashed line: $\sigma^{\eta}=0.005$, solid line: $\sigma^{\eta}=0.010$, dotted line: $\left.\sigma^{\eta}=0.015\right)$. Volatility and distortion are again plotted for increasing price limits (average values of 10 simulation runs, computed in the same way as the solid lines in Fig. 4). While volatility always declines, the reaction of the distortion depends indeed on the intensity of news. To be precise, the higher the variability of the fundamental value, the lower the reduction in the distortion. But even if the average absolute relative change in the fundamental value is $1.2 \%$ per time step $\left(\sigma^{\eta}=0.015\right)$, a modest reduction of mispricing may still be attained. Unfortunately, we do not know the true fundamental volatility of stock markets. However, a standard deviation of $\sigma^{\eta}=0.015$ seems to be quite high.

\section{Conclusions}

Chartists regard volume as an important secondary indicator that confirms price signals: a given price change accompanied by light volume makes the move suspect, whereas it indicates a powerful trading signal when volume is heavy. Guided by such empirical evidence we develop a behavioral stock market model with chartists and fundamentalists. The model has the potential to replicate some important stylized 
facts of stock markets, especially bubbles and crashes, excess volatility, fat tails, uncorrelated price increments, and volatility clustering. The dynamics is mainly driven by the activity of the chartists who tend to trade intensely (mildly) when trading volume is high (low). Using the model as a test bed to investigate the effectiveness of trading breaks we find that trading breaks may indeed calm down stock markets. We observe a reduction in price volatility and, if the fundamental value is not too unsteady, also an improvement of the distortion. The reason is as follows. On the one hand, trading breaks prevent extreme price changes. On the other hand, trading breaks also manage to destroy destabilizing technical pricevolume trading signals.

Of course, more work is needed in order to reach a final assessment of trading breaks. One interesting avenue for future research could be to take into account that trading breaks are likely to reduce market depth. If market depth declines sharply, a given transaction may have a larger price impact than in a more liquid market. Furthermore, if regulators impose restrictions on the trading process, they affect the relative attractiveness of the market. For example, a competing unregulated market may attract additional destabilizing speculators. To conclude, we are convinced that behavioral finance models may improve the understanding of trading restrictions and help regulators to design more efficient markets. Needless to say, this is an important and fascinating field of research. We hope that our paper will stimulate more research in this direction.

\section{Acknowledgments}

Presented at the 13th Annual Symposium of the Society for Nonlinear Dynamics and Econometrics, London, March/April 2005, and at the New Economic Windows Conference, Salerno, September 2004. I thank the participants for helpful

discussions, especially Carl Chiarella, Jerry Coakley, Richard Day, Gulia Iori and Sebastiano Manzan. I also thank an anonymous referee for valuable comments.

\section{References}

[1] W. Brock, J. Lakonishok and B. LeBaron, Simple technical trading rules and the stochastic properties of stock returns, Journal of Finance 67 (1992) 1731-1764.

[2] W. Brock and B. LeBaron, A dynamic structural model for stock return volatility and trading volume, Review of Economics and Statistics 78 (1996) 94-110.

[3] W. Brock and C. Hommes, Heterogeneous beliefs and routes to chaos in a simple asset pricing model, Journal of Economic Dynamics and Control 22 (1998) $1235-1274$.

[4] C. Chiarella, R. Dieci and L. Gardini, The dynamic interaction of speculation and diversification, Applied Mathematical Finance 12 (2005) 17-52.

[5] R. Day and W. Huang, Bulls, bears and market sheep, Journal of Economic Behavior and Organization 14 (1990) 299-329.

[6] P. De Grauwe, H. Dewachter and M. Embrechts, Exchange Rate Theory - Chaotic Models of Foreign Exchange Markets (Oxford, Blackwell, 1993). 
[7] R. Edwards and J. Magee, Technical Analysis of Stock Trends (John Magee, Boston, 1966).

[8] G. Ehrenstein, Cont-Bouchaud percolation model including Tobin tax, International Journal of Modern Physics C 13 (2002) 1323-1331.

[9] E. Fama, Perspectives on October 1987, or, what did we learn from the crash? In Black Monday and the Future of Financial Markets, eds. R. Kampuis, R. Kormendi and J. Watson (Irwin, Homewood, 1989), pp. 71-82.

[10] D. Farmer and S. Joshi, The price dynamics of common trading strategies, Journal of Economic Behavior and Organization 49 (2002) 149-171.

[11] V. France, L. Kodres and J. Moser, A review of regulatory mechanisms to control the volatility of prices, Federal Reserve Bank of Chicago Economic Perspectives 18 (1994) 15-26.

[12] L. Harris, Circuit breakers and program trading limits: What have we learned? In Brookings-Wharton Papers on Financial Services, eds. R. Litan and A. Santomero (Brookings Institutions Press, Washington, 1998), pp. 17-63.

[13] C. Hommes, Financial markets as nonlinear adaptive evolutionary systems, Quantitative Finance 1 (2001) 149-167.

[14] H. Hurst, Long term storage capacity of reservoirs, Transactions of the American Society of Civil Engineers 116 (1951) 770-799.

[15] N. Johnson, P. Jefferies and P. Hui, Financial Market Complexity (Oxford University Press, Oxford, 2003).

[16] D. Kahneman, P. Slovic and A. Tversky, Judgment under Uncertainty: Heuristics and Biases (Cambridge University Press, Cambridge, 1986).

[17] A. Kirman, Epidemics of opinion and speculative bubbles in financial markets, In Money and Financial Markets, ed. M. Taylor (Blackwell, Oxford, 1991), pp. 354-368.

[18] B. LeBaron, Technical trading rule profitability and foreign exchange intervention, Journal of International Economics 49 (1999) 125-143.

[19] A. Lo, H. Mamaysky and J. Wang, Foundations of technical analysis: Computational algorithms, statistical inference, and empirical implementation, Journal of Finance 55 (2000) 1705-1765.

[20] Y.-H. Lui and D. Mole, The use of fundamental and technical analysis by foreign exchange dealers: Hong Kong evidence, Journal of International Money and Finance 17 (1998) 535-545.

[21] T. Lux, Herd behavior, bubbles and crashes, Economic Journal 105 (1995) 881-896.

[22] T. Lux, The limiting extremal behavior of speculative returns: An analysis of intradaily data from the Frankfurt stock exchange, Applied Financial Economics 11 (2001) 299-315.

[23] T. Lux and M. Marchesi, Volatility clustering in financial markets: A micro-simulation of interacting agents, International Journal of Theoretical and Applied Finance 3, (2000) 675-702.

[24] T. Lux and M. Ausloos, Market fluctuations I: Scaling, multiscaling, and their possible origins, in Science of Disaster: Climate Disruptions, Heart Attacks, and Market Crashes, eds. A. Bunde, J. Kropp and H. Schellnhuber (Springer, Berlin, 2002), pp. 373-410.

[25] R. Mantegna and E. Stanley, An Introduction to Econophysics (Cambridge University Press, Cambridge, 2000).

[26] L. Menkhoff, Examining the use of technical currency analysis, International Journal of Finance and Economics 2 (1997) 307-318. 
[27] J. Murphy, Technical Analysis of Financial Markets (New York Institute of Finance, New York, 1999).

[28] M. Pring, Technical Analysis Explained (McGraw-Hill, New York, 1991).

[29] H. Simon, A behavioral model of rational choice, Quarterly Journal of Economics 9 (1955) 99-118.

[30] V. Smith, Papers in Experimental Economics (Cambridge University Press, Cambridge, 1991).

[31] J. Sonnemans, C. Hommes, J. Tuinstra and H. van de Velden, The instability of a heterogeneous cobweb economy: A strategy experiment on expectation formation, Journal of Economic Behavior and Organization 54 (2004) 453-481.

[32] D. Sornette, Why Stock Markets Crash (Princeton University Press, Princeton, 2003).

[33] M. Taylor and H. Allen, The use of technical analysis in the foreign exchange market, Journal of International Money and Finance 11 (1992) 304-314.

[34] F. Westerhoff, Speculative markets and the effectiveness of price limits, Journal of Economic Dynamics and Control 28 (2003) 493-508.

[35] F. Westerhoff, Heterogeneous traders and the Tobin tax, Journal of Evolutionary Economics 13 (2003) 53-70.

[36] F. Westerhoff, Multiasset market dynamics, Macroeconomic Dynamics 8 (2004) 596-616.

[37] C. Wieland and F. Westerhoff, Exchange rate dynamics, central bank interventions and chaos control methods, Journal of Economic Behavior and Organization 58 (2005) 117-132. 